Int. J. Electrochem. Sci., 14 (2019) $4173-4184$

\title{
Studies on the Effect of the Substrate on the Electrocatalytic Performance of Electrodeposited NiFe Hydroxides for Oxygen Evolution Reaction
}

\author{
Hansheng $\mathrm{Wei}^{2}$, Jie Liu ${ }^{1, *}$, Yida Deng ${ }^{1}$,Wenbin $\mathrm{Hu}^{1}$ and Cheng Zhong ${ }^{1, *}$ \\ ${ }^{1}$ School of Materials Science and Engineering, Tianjin University, Tianjin 300072, China \\ ${ }^{2}$ Fujian Jianou First High School, Fujian 353100, China \\ *E-mails: jieliu0109@ tju.edu.cn; cheng.zhong@tju.edu.cn
}

doi: $10.20964 / 2019.05 .45$

Received: 4 January 2019 / Accepted: 28 February 2019 / Published: 10 April 2019

\begin{abstract}
As one of the most promising candidates for the oxygen evolution reaction (OER), NiFe hydroxides have attracted tremendous attention due to their earth abundance, cost-effectiveness, and relatively high catalytic activity. In this work, to investigate the effect of the underlying substrate on the electrocatalytic activity of $\mathrm{NiFe}$ hydroxides for the OER, NiFe hydroxides were prepared on various substrates including $\mathrm{Au}, \mathrm{Cu}, \mathrm{C}$, ITO, and Ti via an electrodeposition technique. The whole preparation process is extremely clean since it avoids the introduction of any surfactants, binders, reducers or capping agents, ensuring the clean surface of NiFe hydroxides and thus allowing a true comparison of the effect of the substrate composition on the electrocatalytic performance of NiFe hydroxides. The surface morphology and chemical element distribution of the electrodes were studied by a scanning electron microscope and energy dispersive $\mathrm{X}$-ray analysis, and the chemical composition was characterized by X-ray photoelectron spectroscopy. The NiFe hydroxides electrodeposited on various substrates exhibit similar thin film surface morphologies and are grown horizontally on the surface of the substrate, possessing some small wrinkles approximately vertically aligned to the surface. Among the NiFe hydroxides formed on the various substrates, the NiFe hydroxides on the Au substrate exhibit the most remarkable enhancement in electrocatalytic activity towards the OER, and the current density reaches $149 \mathrm{~mA} \mathrm{~cm}^{-2}$ at $0.8 \mathrm{~V}$ (vs. saturated calomel electrode). The catalytic current density shows no evident decay after an accelerated durability test of $10000 \mathrm{~s}$. This work provides a systematic study on the influence of the substrate composition on the electrocatalytic activity of electrodeposited NiFe hydroxides and demonstrates new ways to develop high-performance catalysts.
\end{abstract}

Keywords: NiFe hydroxides; electrodeposition; substrate composition; oxygen evolution reaction 
(C) 2019 The Authors. Published by ESG (www.electrochemsci.org). This article is an open access article distributed under the terms and conditions of the Creative Commons Attribution license (http://creativecommons.org/licenses/by/4.0/). 